\title{
The Cylindrical-GEM detectors for the KLOE-2 Inner Tracker
}

\section{G. Bencivenni* Laboratori Nazionali di Frascati - INFN, Frascati, Italy}

E-mail: giovanni.bencivenni@lnf.infn.it

A. Balla, S. Cerioni, P. Ciambrone, E. De Lucia, D. Domenici, J. Dong, G. Felici, M. Gatta, G. Morello, A. Palladino, M. Poli Lener, E. Tskhadadze

Laboratori Nazionali di Frascati - INFN, Frascati, Italy

G. De Robertis, G. Fanizzi, N. Lacalamita, R. Liuzzi, F. Loddo, M. Mongelli, A. Ranieri, V. Valentino

INFN Sezione di Bari - Bari, Italy

\section{Capodiferro, A. Di Domenico, A. Pelosi}

Dipartimento di Fisica, "Sapienza" Università di Roma and INFN Sezione di Roma - Roma, Italy

\section{P. Branchini, A. Budano}

INFN Sezione di Roma Tre - Roma, Italy

\section{E. Czerwinski}

\section{Jagiellonian University, Institute of Physics - Krakow, Poland}

Part of the upgrade of the KLOE detector at the DAФNE $\Phi$-factory consists of the insertion of a tracking device around the interaction region, composed of four tracking layers with radii from $130 \mathrm{~mm}$ to $205 \mathrm{~mm}$ and an active length of $700 \mathrm{~mm}$. Each layer was realized as a cylindrical triple-GEM (CGEM) kapton-based detector, a solution that allows us to keep the total material budget below $2 \%$ of $X_{0}$, of utmost importance to limit the multiple scattering of low-momentum tracks at KLOE-2, and to minimize dead spaces. The peculiar readout pattern with XV strips provides a spatial resolution of about $200 \mu \mathrm{m}$ on both views, while a dedicated readout system has been developed by the KLOE-2 collaboration. It is composed of a digital readout front-end card based on the GASTONE ASIC and a General Interface Board with a configurable FPGA architecture and Gigabit Ethernet. The construction of the four CGEM layers has been completed and the detectors have been tested with a beta source and cosmic-ray muons. The insertion inside the KLOE apparatus was performed in July 2013. The construction procedure and the results of the validation tests will be reported.

52 International Winter Meeting on Nuclear Physics - Bormio 2014,

27-31 January 2014

Bormio, Italy

\footnotetext{
* Speaker.
} 


\section{The KLOE experiment}

The KLOE experiment located at the Frascati $\phi$-factory, DA $\Phi N E$, achieved many important results in kaon and hadronic physics [1]. A new data taking period is going to start with an upgraded collider which will allow the physics program to cover neutral kaon interferometry, $K_{S}, \eta$ and $\eta^{\prime}$ rare decays and searches for exotic particles [2]. To this purpose the reconstruction performance for tracks near the Interaction Point (IP) has been improved by the installation of an inner tracker, based on cylindrical GEM (Gas Electron Multiplier [3]) technology. It was installed in the region between the beam pipe (radius $100 \mathrm{~mm}$ ) and the Drift Chamber (inner wall radius $250 \mathrm{~mm}$ ). GEM technology has been used in forward regions of experiments at hadron machines (COMPASS, LHCb, TOTEM) because of its excellent rate capability, but KLOE-2 will be the first experiment ever to use four coaxial CGEM layers. The KLOE-2 experiment requires this detector to be extremely low mass to limit the multiple scattering of low-momentum tracks, photon conversion and $K_{S}$ regeneration.
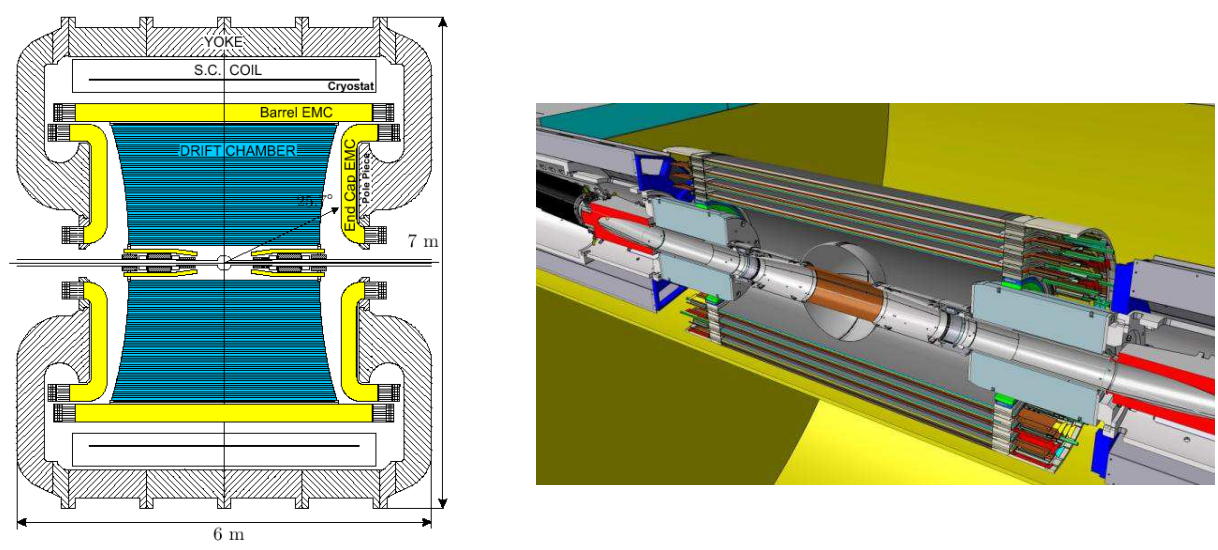

Figure 1: The KLOE apparatus (left) and the position of the Inner Tracker around the beam pipe (right).

\section{Detector description}

The performances of the KLOE Drift Chamber, operated in a $0.5 \mathrm{~T}$ axial magnetic field, can be summarized with a spatial resolution of $\sigma_{r, \varphi} \simeq 150 \mu \mathrm{m}$ in the bending plane and $\sigma_{z} \simeq 2 \mathrm{~mm}$ along the beam line, leading to a $\sigma\left(p_{\perp}\right) / p_{\perp} \sim 0.4 \%$. In order to minimize the multiple scattering of low-momentum tracks the Inner Tracker (IT) matches a total material budget of $2 \% X_{0}$, providing spatial resolutions of $\sigma_{r, \varphi} \sim 200 \mu \mathrm{m}$ and $\sigma_{z} \sim 500 \mu \mathrm{m}$ inside the $0.5 \mathrm{~T}$ magnetic field [4]. The addition of four points towards the IP will improve about a factor 3 the vertex resolution of $\mathrm{K}_{S} \rightarrow \pi \pi$, presently limited to $\sim 2 \mathrm{~mm}$. The KLOE-2 Inner Tracker is composed of four coaxial layers with radii between $130 \mathrm{~mm}$ and $205 \mathrm{~mm}$, and an active length of $700 \mathrm{~mm}$. The inner radius was chosen to be $120 \mathrm{~mm}=20 \tau_{\mathrm{S}}$ in order to preserve the $K_{L}-K_{S}$ quantum interference region [5]. Such dimensions motivated the CERN TE-MPE-EM workshop, producing the GEM foils, to develop a new manufacturing process called the "single mask technique" [6]. Each cylindrical GEM electrode is composed of three foils, each of them segmented into 40 sectors on the top side 


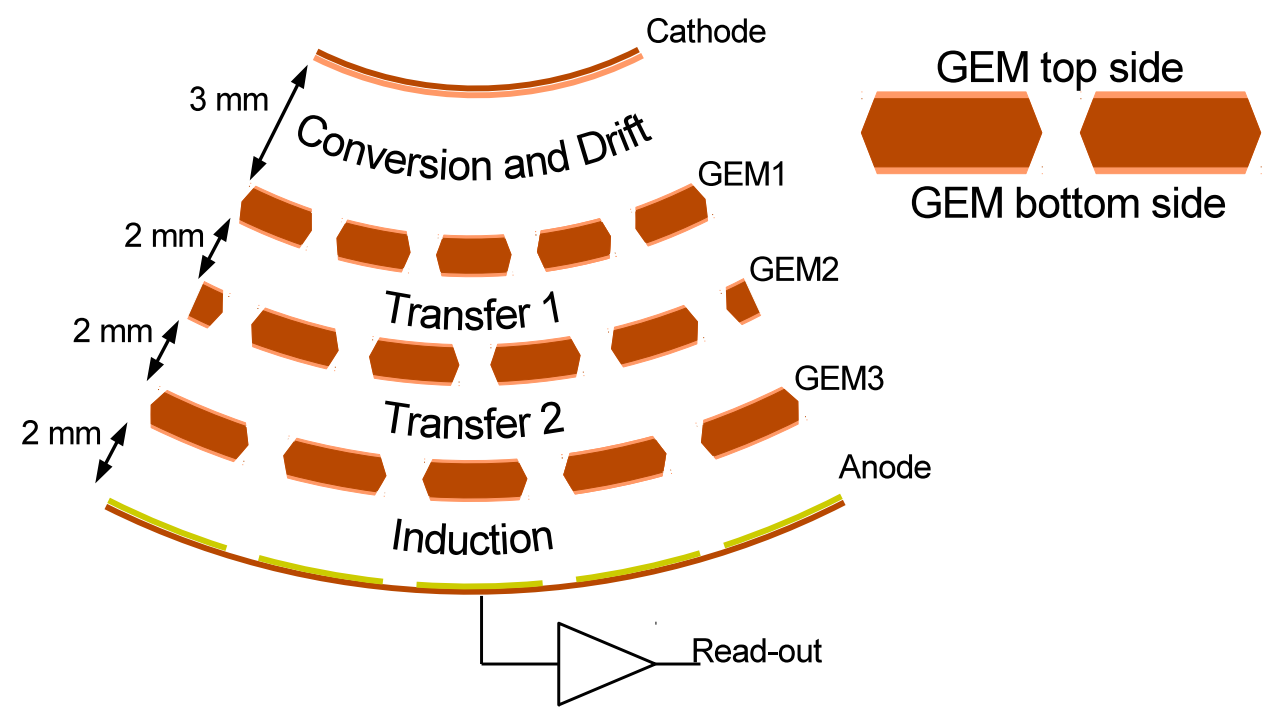

Figure 2: Sketch of the cross section of a cylindrical GEM with the conventional terms to identify the volumes and surfaces of a triple-GEM.
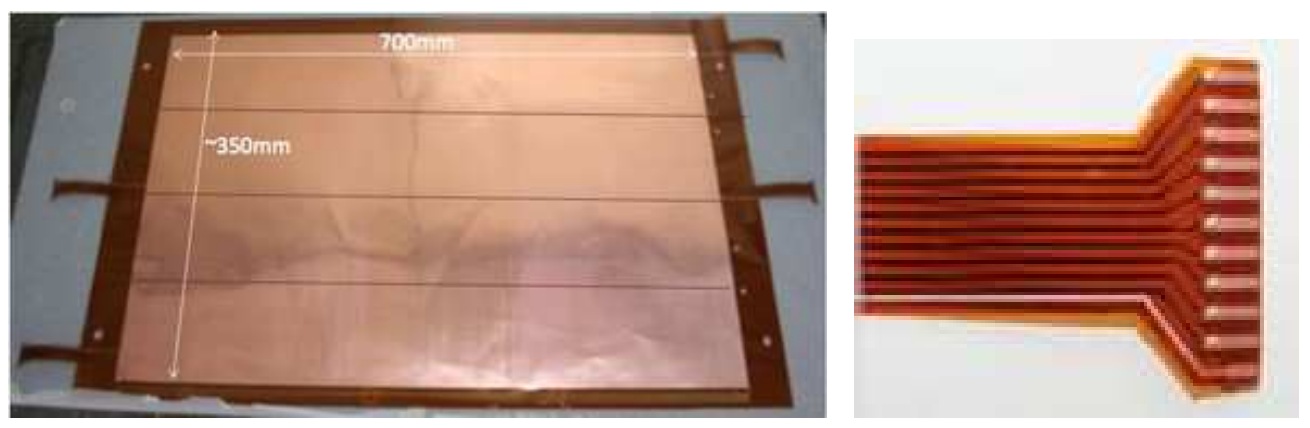

Figure 3: Picture of a large GEM foil with evidence of the HV segmentation (left) and detail of HV tail (right).

and 4 sectors on the bottom side (Figure 2), independently supplied with high voltage (HV), in order to reduce the energy of possible discharges. The HV connector is soldered on the bottom face of each tail present on the sides of the foil (Figure 3 (left)). The connector has eleven channels linked to the active area by copper strips: ten for the sectors on the top side and one for the sector on the bottom side. The former ones are jointed to the connector, on the opposite side of the foil, through holes (vias) filled with conductive glue (Figure 3 (right)). The readout plane is a flexible kapton/copper circuit produced in the same workshop as the GEM foils.

As shown in Figure 4 the readout is patterned with $X$ strips, parallel to the CGEM axis providing the coordinate on the $r-\varphi$ plane. The parallelogram shaped pads are connected by vias to a backplane formed by strips ( $V$ strips) forming an angle with respect to the $X$ strips. 

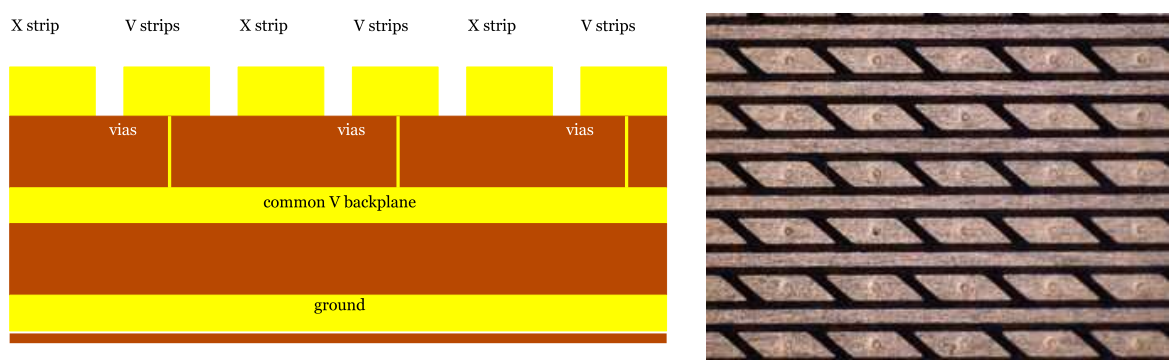

Figure 4: Readout scheme (left) and microscopic picture of a readout plane (right).

\subsection{Front-end Electronics}

The strips are read-out by a front-end board hosting two GASTONE ASIC chips [7]. Each chip has 64 channels featuring low equivalent noise $\left(800 \mathrm{e}^{-}+40 \mathrm{e}^{-} / \mathrm{pF}\right)$ and low power consumption ( $\sim 200 \mathrm{~W}$ for the 30000 total channels of the IT) composed of four different stages: a charge preamplifier ( $20 \mathrm{mV} / \mathrm{fC}$ sensitivity), a shaper, a leading-edge discriminator with programmable threshold, and a monostable which stretches the digital signal in order to be synchronized with the KLOE Level 1 trigger. The DAQ chain is composed of a General Interface Board (GIB) and a readout driver (ROD) [8]: the former is based on a Xilinx Virtex 4FX Filed Programmable Gate Array (FPGA) with an embedded IBM Power PC (PPC405) running at $300 \mathrm{MHz}$. Each GIB supplies power to 16 GASTONE chips from which they download data [9]. The ROD performs the first stage of event-building collecting data from GIBs through their $2 \mathrm{~Gb} / \mathrm{s}$ optical port. The ROD then sends its data to the online computing farm [10].

\section{Construction details}

\subsection{Quality tests}

The quality of the GEM foils are first checked by optical inspection [11] to find any macroscopic defects and then by measuring of the resistance present on the vias of the HV tail (Figure 3 (right)) where the HV connectors are soldered (the resistance must be lower than $2 \Omega$ ). Each GEM sector is then supplied with up to $600 \mathrm{~V}$ in a $\mathrm{N}_{2}$-flushed box to keep the relative humidity below $10 \%$. The discharge rate, $R_{D}$, and dark current, $I_{D}$, are measured. The quality acceptance threshold on the current requires $I_{D}<1 \mathrm{nA}$. During the test the maximum measured discharge rate was $R_{D} \leq 3$ discharges / hour.

The quality test of the readout was performed by the time domain reflectometer method [12]: pulsing each strip with a signal whose decay time is a function of the strip length, it's possible to detect any damage (including bad soldering of the connectors) or short-circuits.

\subsection{Construction}

The construction procedure of each cylindrical electrode is similar for GEM. Three foils are fixed in position on a precisely machined assembly table by reference pins and spliced together (Figure 5 (top left)) using an epoxy along their larger edges. The overlap is about $3 \mathrm{~mm}$. The curing cycle of the epoxy takes place in a $\sim 0.9$ bar vacuum-bag. This very large foil is rolled on 
a precisely machined aluminum mould with a $400 \mu \mathrm{m}$ thick teflon surface. Two circular fiberglass frames are glued aside the active area of the cylindrical electrode (Figure 5 (bottom left)): these frames act as mechanical supports and as spacers between two consecutive electrodes. The cylindrical cathode is reinforced on its innermost surface with a $3 \mathrm{~mm}$-hick Nomex honeycomb, while the readout is laminated with two $90 \mu \mathrm{m}$ thick carbon-fiber skins interleaved by a $5 \mathrm{~mm}$-thick Nomex honeycomb. For the two outermost CGEM electrodes a spacing grid was introduced in the gas gaps to keep the distance between the GEMs even in case of mechanical relaxation. Each grid was assembled with 12 rods and 8 rings realized from $300 \mu \mathrm{m}$ thick Peek pieces. The electrodes
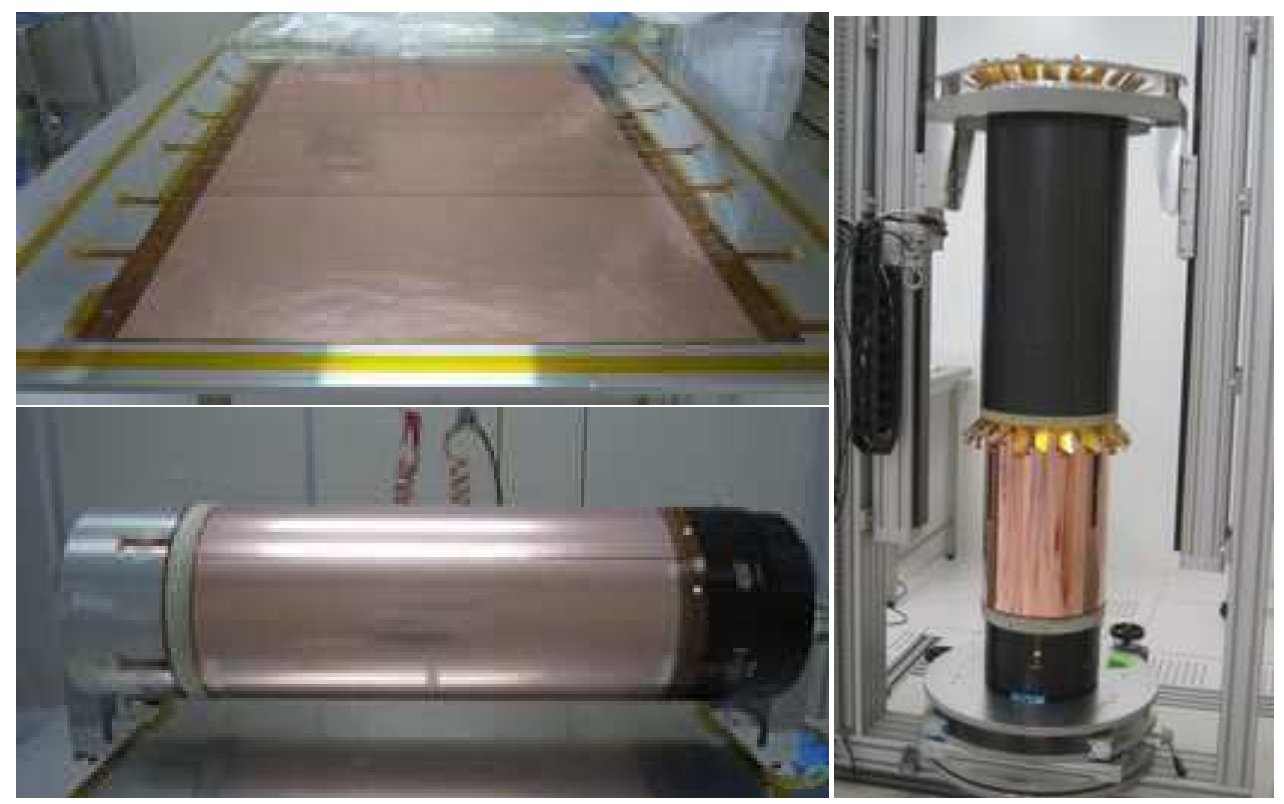

Figure 5: Steps of a cylindrical GEM realization: large planar foil (top left), cylindrical electrode (bottom left) and layer assembly (right).

are then inserted one into the other using a Vertical Insertion Machine (Figure 5 (right)) providing a precision on the alignment between the two axes of $\sim 100 \mu \mathrm{m}$ over $1 \mathrm{~m}$ length. The machine allows the rotation of the mould for the detector sealing, done with the same epoxy used to glue the foils.

\section{Tests of the layers}

The first test of all the layers was to check the gas tightness. The outgoing gas flux was measured to be $\sim 99 \%$ of the ingoing flux. Then each CGEM layer was tested with a ${ }^{90} \mathrm{Sr}$ source positioned at the center of each $\mathrm{HV}$ sector of the GEM foils and with cosmic-ray muons. The detectors were flushed with an $\mathrm{Ar} \mathrm{iC}_{4} \mathrm{H}_{10} 90: 10$ gas mixture. The use of an isobutane-based gas mixture allows a large reduction of the discharge occurence in the detector with respect to $\mathrm{Ar}: \mathrm{CO}_{2}$ (Figure 7 (right)). The gain of a triple-GEM depends on the sum of the voltage applied to the three GEM foils: for the chosen gas mixture a sum of $V=860 \mathrm{~V}$ corresponds to a gain of $\sim 2 \cdot 10^{4}$ (Figure 7 (left)) matching the front-end electronics performance. The electric fields needed to transport the charge between two consecutive GEM foils are set to $1 / 1.5 / 1.5 / 5 \mathrm{kV} / \mathrm{cm}$. The test with a movable 


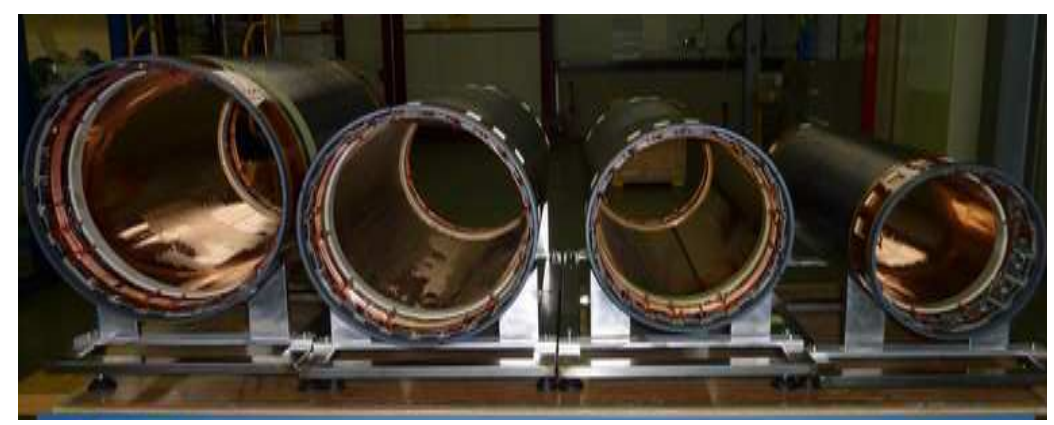

Figure 6: The four layers composing the KLOE-2 IT
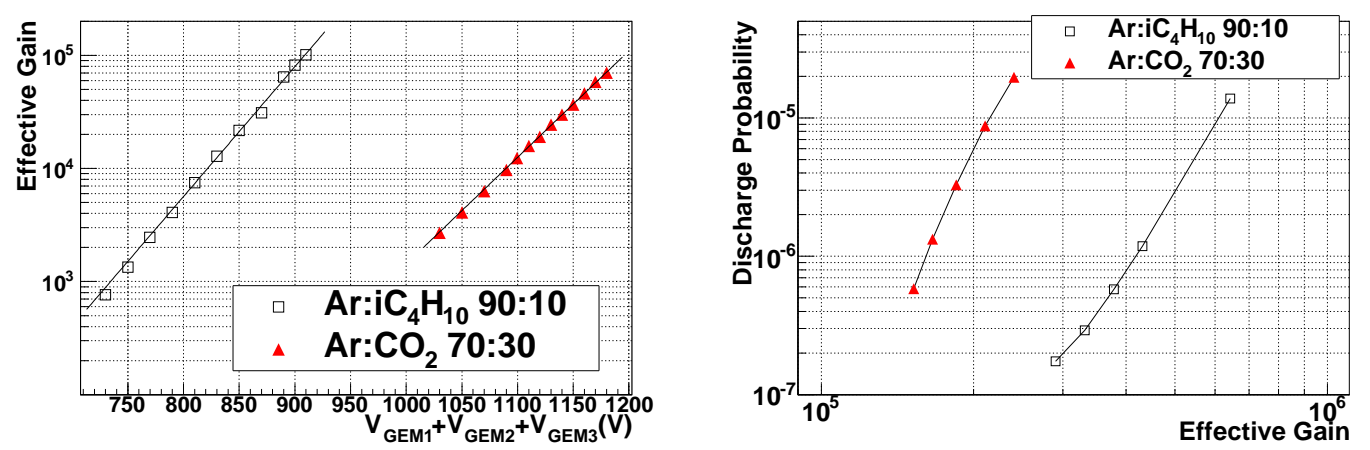

Figure 7: Measurements with two gas mixtures: gain as a function of the voltage applied on the GEMs (left) and discharge rate as a function of the gain (right).

${ }^{90} \mathrm{Sr}$ source (Figure 10 (left)) was performed to check the uniformity of the detector response and the final HV distribution and signal cables. The source test showed evidence of a correlated noise (Figure 9 (left)). This effect, already observed from the LHCb collaboration, can be explained as a capacitive coupling between GEM3 bottom side and the readout plane (Figure 8). This correlated noise can be strongly reduced by implementing an RC-circuit between the GEM3 bottom side and the ground plane of the readout (Figure 4). Studies on the optimization of the $R$ value are reported in Figure 9 (right). The number of hits collected on the $X$-strips was recorded for different values of the resistance. The cross-talk was defined as

$$
\mathrm{X}-\operatorname{talk}(R) \equiv \frac{\int_{S_{x}} H\left(s_{x}, R\right) \mathrm{d} s_{x}-\int_{S_{x}} H\left(s_{x}, R_{\min }\right) \mathrm{d} s_{x}}{\int_{S_{x}} H\left(s_{x}, R_{\min }\right) \mathrm{d} s_{x}}
$$

where $S_{x}$ is the set of $\mathrm{X}$-strips illuminated by the source, $H\left(s_{x}, R\right)$ is the number of hits for each $\mathrm{X}$-strip at a given $R$ and $R_{\min }$ is the value of the resistor that minimizes the integral. The decoupling of the GEM3 bottom HV sectors from their facing readout foils has been done chosing $R=200 \Omega$ and $C=2.2 \mathrm{nF}$ for all the layers.

For the cosmic-ray muon test the coincidence of the signals coming from two scintillators provided the data acquisition trigger, while the three planar GEM chambers were used for the reference tracking system. Figure 10 (right) shows, for layer 3, the hits distribution of the clusters selected requiring a track reconstructed by the three GEM chambers. The hits are projected on the unrolled readout plane $\rho-\xi$, where $\xi$ is directed along the CGEM axis (z axis in KLOE reference frame). 


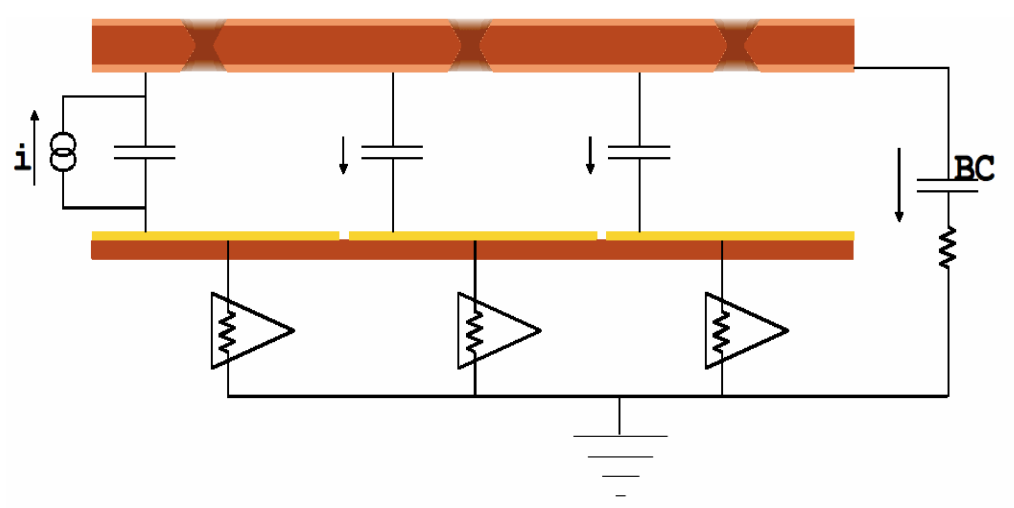

Figure 8: Schematic diagram of the Blocking Capacitor.
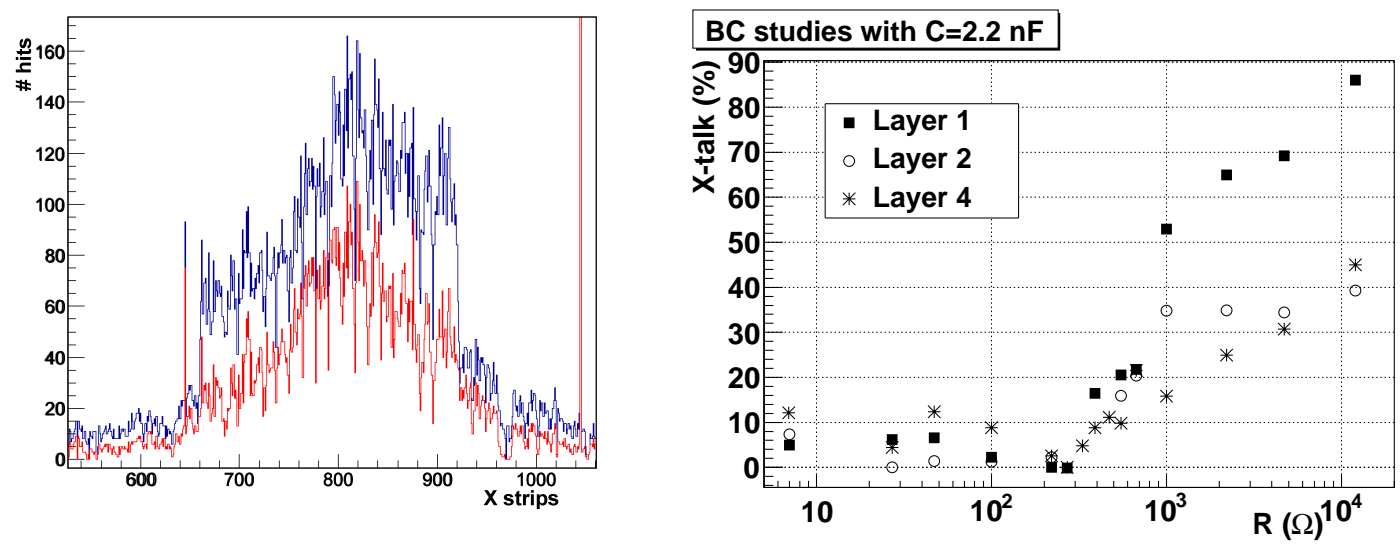

Figure 9: An example of correlated noise (left) and its dependence on the resistance, $R$, for three of the IT layers (right).

Unlike the $\beta$ source test, where one only cluster distributions is visible, in this case we have two populations per layer since muons cross twice a CGEM. The spatial resolution is computed from the distribution of the residuals: the distance between the track reconstructed by the reference tracking system and the response from the CGEM. A very preliminary distribution of the residuals along the $\xi$ coordinate obtained with cosmic-ray muon data is shown in Figure 11. A spatial resolution of $\sim 600 \mu \mathrm{m}$ was obtained, not so far from our target, due to systematic effects under study. Along $\rho$ the spatial resolution can be computed, because of the digital readout, as the ratio of the X-strips pitch to $\sqrt{12}$ without magnetic filed. A measurement of the spatial resolution on the direction orthogonal to the X-strips with the same pitch in a $0.5 \mathrm{~T}$ magnetic field is reported in [16], showing a $\sigma_{\rho} \sim 200 \mu \mathrm{m}$, in agreement with the requirement from KLOE-2 collaboration.

\section{IT integration}

The IT was finally assembled on 14 March 2013 and it was equipped with copper coils for the cooling of the front-end electronics. Two additional air inflating systems were installed in front of the IT end caps and between the space enclosed by the beam pipe and the innermost layer of the 

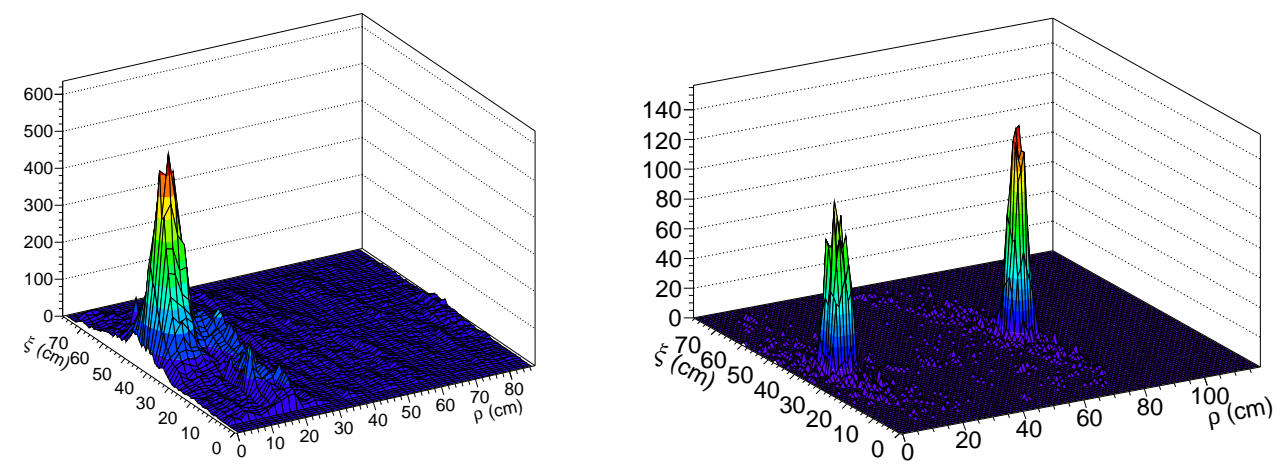

Figure 10: Clusters reconstructed in a CGEM layer in the unrolled readout plane $(\rho-\xi): \beta$ source (left) and cosmic-ray muons (right).

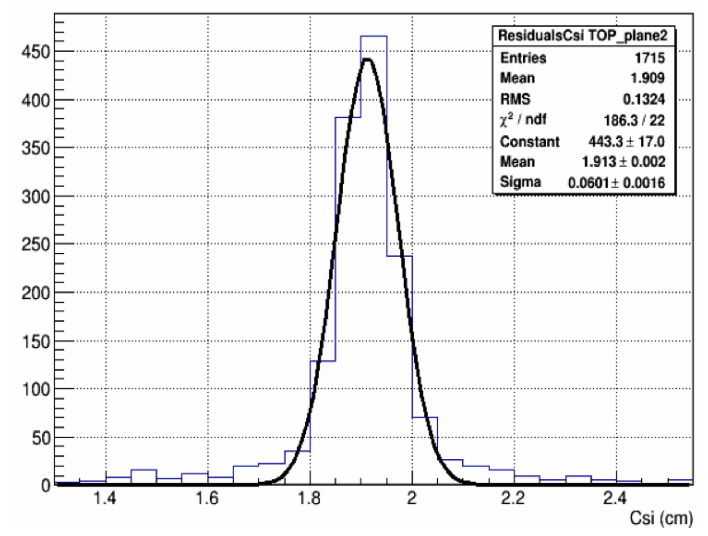

Figure 11: Residuals of the tracks reconstructed with the PGEMs and the hits recorded by Layer 3

IT. After the positioning in the DA $\Phi N E$ interaction region the detector was tested with a pulsing system and cosmic-ray muons to check the detector cabling and operational conditions.

\section{Conclusions}

The KLOE-2 collaboration is commissioning the new sub-detectors required to accomplish a new physics program focused on decay channels very close to the interaction point. The requirements for the upgrade of the tracking system have been fullfilled using the cylindrical GEM detectors, which pushed for a new manufacturing technique developed by the CERN TE-MPE-EM workshop. The tests on each of the four triple-GEM layers composing the KLOE-2 Inner Tracker have shown good operational stability. The IT integration in the DA $\Phi$ NE interaction region was completed in July 2013. The optimization of the operational parameters, the alignment and the calibration of the detector are part of the commissioning program which is ongoing. 

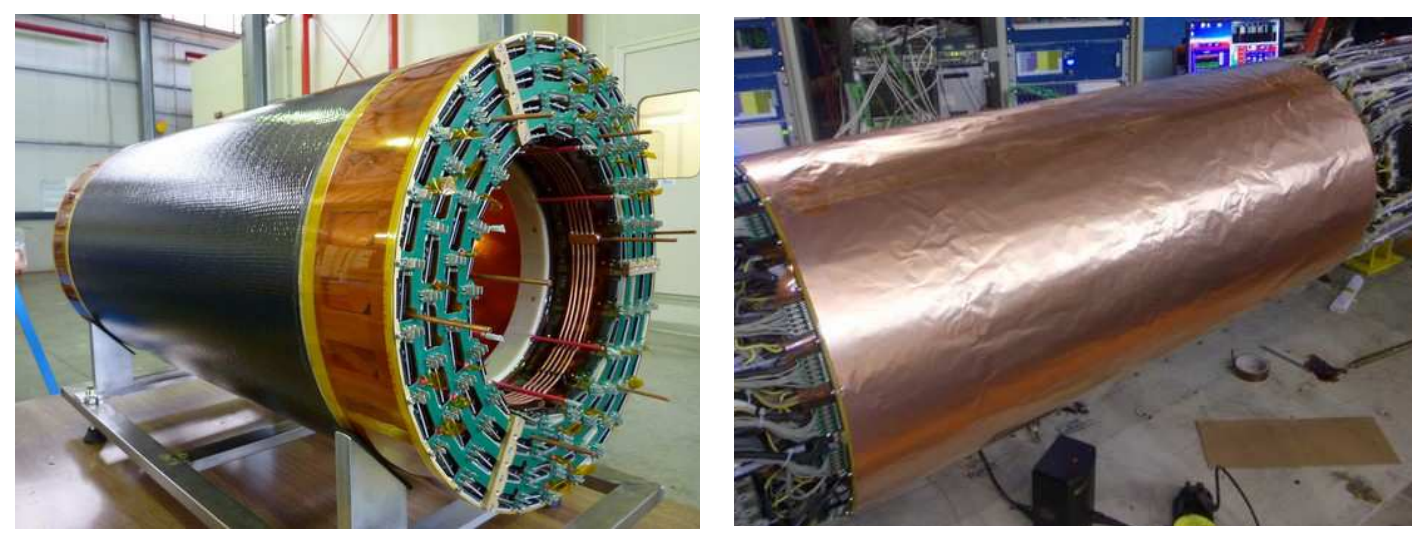

Figure 12: The KLOE-2 Inner Tracker (left) and its final position on the DAФNE Interaction Region.

\section{References}

[1] F. Bossi et al. and KLOE collaboration, Precision Kaon and Hadron Physics with KLOE, Rivista del Nuovo Cimento 31 (2008).

[2] G. Amelino Camelia et al., Physics with the KLOE-2 experiment at the upgraded DAФNE, Eur. Phys. J C 68619 (2010).

[3] F. Sauli, GEM: A new concept for electron amplification in gas detectors, Nucl. Inst. \& Meth. A 386 (1997) 531.

[4] A. Balla et al., Design and Construction of a cylindrical GEM detector as the Inner Tracker device of the KLOE-2 experiment, in proceedings of 8th International Conference on Nuclear Physics at Storage Rings-Stori11, October 9-14, 2011 Laboratori Nazionali di Frascati dell'INFN, Italy POS (STORI11) 071.

[5] KLOE-2 collaboration, TDR of Inner Tracker for KLOE-2 experiment LNF-10/3(P) INFN-LNF, Frascati (2010) and arXiv:1002.1557v1.

[6] M. Alfonsi et al., Activity of CERN and LNF groups on large area GEM detectors, Nucl. Inst. \& Meth. A 617 (2010) 151.

[7] A. Balla et al., GASTONE: a new ASIC for the cylindrical GEM Inner TRacker of KLOE experiment at $D A \Phi N E$, Nucl. Inst. \& Meth. A 604 (2009) 23.

[8] P.Branchini et al., Front-end DAQ strategy and implementation for the KLOE-2 experiment, 82013 T04004.

[9] E. De Lucia et al., Development of CGEM technology for ultra-light tracking detectors: the KLOE-2 Inner Tracker, to be published on Acta Physics Polonica B.

[10] A. Aloisio et al., Data acquisition and monitoring for the KLOE detector, Nucl. Inst. \& Moth. A 516 (2004) 288.

[11] A. Balla et al., Construction and test of the Cylindrical-GEM detectors for the KLOE-2 Inner Tracker, to be published on Nucl. Instr. \& Meth. A.

[12] G. Bencivenni et al., A Time Domain Reflectometer with 100 ps precision implemented in a cost.effective FPGA for the test of the KLOE-2 Inner Tracker readout anodes, Nucl. Inst. \& Meth. A 698 (2013) 185. 
[13] A. Balla et al., Status of the cylindrical-GEM project for the KLOE-2 inner tracker, Nucl. Instr. \& Meth. A 628 (2011) 194-198.

[14] G. Bencivenni et al., The full scale prototype of the cylindrical-GEM detector as inner tracker in KLOE-2, "Nuclear Symposium Conference Record", 2007 IEEE, pp 4666-4670.

[15] A. Balla et al., Performance of the cylindrical-GEM prototype for the inner tracker of KLOE-2, "Nuclear Symposium Conference Record", 2008 IEEE, pp 1345-1347.

[16] A. Balla et al., Production and test of the first triple-GEM cylindrical layers of the KLOE-2 Inner Tracker, "Nuclear Symposium Conference Record", 2012 IEEE, pp 754-758. 